\title{
Medication-Related Osteonecrosis of the Jaw and Low-Level Laser Therapy as Adjuvant Treatment: A Case Report
}

\author{
Amanda Azevedo Torres ${ }^{1 *}$, Beatriz Leal de Freitas², Patrick Parry Carneiro², André Luca Araujo de Sousa², \\ Maria Ângela Arêa Leão Ferraz ${ }^{3}$, Jean de Pinho Mendes ${ }^{4}$, André Luiz Ferreira Costa ${ }^{5}$, Antonione Santos \\ Bezerra Pinto ${ }^{6}$ \\ 'Undergraduate Medical Student at Federal University of Piauí, Parnaíba, Brazil \\ ${ }^{2}$ Undergraduate Dental Student at the State University of Piauí, Parnaíba, Brazil \\ ${ }^{3} \mathrm{PhD}$, Dentistry Professor at the State University of Piauí, Teresina, Brazil \\ ${ }^{4} \mathrm{PhD}$ Student and Dentistry professor at the State University of Piauí, Parnaíba, Brazil. \\ ${ }^{5}$ Postgraduate Program in Dentistry - Cruzeiro do Sul University (UNICSUL) - São Paulo, Brazil \\ ${ }^{6} \mathrm{PhD}$ Student in Morphofunctional Sciences and Medical Professor at Faculty of Human, Exact and Health Sciences of \\ Piauí of the Higher Education Institute of the Vale do Parnaíba, Parnaíba, Brazil
}

*Correspondence to
Amanda Azevedo Torres, Email amandator@live.com

Published online October 3, 2020

\begin{abstract}
Introduction: Medication-related osteonecrosis of the jaw (MRONJ) occurs by the use of a drug that has osteonecrosis as one of its side effects.

Case Report: We describe a case of a 48-year-old oncological patient who had brain and bone metastasis due to breast cancer and was medicated with bisphosphonates (BPs). She presented cavities, and after an incorrect exodontia, the lesion evolved into a jaw osteonecrosis. Then she did a sequestrectomy and was treated using laser therapy. Radiological and clinical features are also described.

Conclusion: In a case like this, we notice how necessary is a complete evaluation of the oncological patient before some procedures and laser therapy as an effective ally in the management.

Keywords: Bisphosphonate-associated osteonecrosis of the jaw; Laser therapy; Low-level Light therapy.
\end{abstract}

\section{Introduction}

The osteonecrosis of the jaws was first described as a consequence of radiotherapy. However, it was presented as a result of the uninterrupted use of medicines. Bisphosphonates (BP), for instance, are the most frequent medications associated with medication-related osteonecrosis of the jaw (MRONJ). ${ }^{1}$

BPs are drugs that inhibit bone resorption and are used to treat bone disorders such as osteoporosis, osteopenia, Paget's disease, and metastatic bone disease. They make the bone renovation difficult by suppressing the recruitment and activity of osteoclasts, shortening their life cycle, and having antiangiogenic properties. Depending on the time of treatment or the administered drug, the BP can remain in the body for years and it can cause ischemia and develop osteonecrosis, a rare but serious collateral effect. $^{2-4}$

As a result of chemotherapy or associated medications, another frequent oral complication is mucositis, the most common cause of oral pain during treatment. ${ }^{5}$

Necrosis in patients using BP after a dental procedure presents a risk to the patient's health, and therefore it must be analyzed by an oral surgery specialist with a physician to choose the best time for the procedure, considering that MRONJ implies the reduction of the quality of life and significant morbidity in the affected ones, ${ }^{6}$ while the early diagnosis can reduce the morbidity resulting from the lesions. Then, during the treatment with BP, the patient might have good oral health and all invasive procedures should be avoided.?

In this case report, we present a case of MRONJ owing to its importance in clinical practice and the laser therapy as an ally for the treatment and the evolution evidenced, aiming to understand its clinical presentation.

\section{Case Presentation}

A 48-year-old woman had a previous history of cancer on her right breast in 2012, with six sessions of chemotherapy.

Please cite this article as follows: Torres AA, de Freitas BL, Carneiro PP, de Sousa ALA, Arêa Leão Ferraz MA, de Pinho Mendes J, et al. Medication-related osteonecrosis of the jaw and low-level laser therapy as adjuvant treatment: a case report. J Lasers Med Sci. 2020;11(4):497-499. doi:10.34172/jlms.2020.78. 
Five years later, surgical procedures were performed combining a mastectomy and 28 radiotherapy sessions. She did not mention any dental evaluation before the treatment with Cisplatin and Gemcitabine had started.

Next year, bone metastasis was detected and, as a result, BP intravenous therapy started (Zoledronic acid). In 2015 , due to cavities with pulp exposure, an extraction of 46 th, 47 th and 48 th teeth were done. However, the patient complaining of latent pain in that area returned to the same professional so periapical radiograph in the molar region was taken, which showed a radiolucent image in the area corresponding to tooth 47 , compatible with recent extraction and presenting bone irregularity in the alveolar crest. The occlusal radiograph of the jaw observed discreet periosteal reaction projected at inferior cortical (basilar area), suggesting osteomyelitis. The panoramic radiograph (Figure 1D) showed a heterogeneous image projected in the right region in the jaw with sclerotic reaction and bone sequestrum (irregular opacity) since there was inflammation/infection process in the bone.

Unfortunately, the patient did not return after doing the examinations and just went to another dentist. In clinical examination, the right area of the jaw body was very swollen; 46, 47 and 48 elements were absent and had defective alveolar repair with bone exposure and active purulent secretions. Therefore, the treatment administered was clindamycin, orally, and mouth rinses with chlorhexidine in $0,12 \%$ and with $\mathrm{H}_{2} \mathrm{O}_{2}$. The culture with an antibiogram showed it was coagulase-negative Staphylococcus ciprofloxacin-sensitive so clindamycin antibiotic therapy was maintained and acetaminophen was added for pain control.

A sequestrectomy was performed with general anesthesia and the necrotic bone was removed and debrided, although was not possible to do a reconstruction with adequate coverage of soft tissues on the exposed area, which had influenced the final result. Some samples were analyzed macroscopically as multiple hard tissue fragments, measuring $15 \mathrm{~mm}$ x $09 \mathrm{~mm} \times 05 \mathrm{~mm}$ together, size and surface irregulars, brownish, blackish color and stone consistency. On microscopic examination, the demineralized histological sections revealed a fragment of lamellar bone tissue showing gaps without osteocytes. In the bone tissue periphery, large areas of resorption were observed. Regions with intense inflammatory infiltrate consisting of polymorphonuclear cells were observed with the presence of bacterial colonies. The histopathological diagnosis was osteonecrosis in the right posterior region of the mandible.

Two months later, the low-level laser therapy (LLLT) started with Therapy EC Equipment at $\lambda 660 \mathrm{~nm} \pm 10 \mathrm{~nm}$ (red spectrum) with fixed power of $100 \mathrm{~mW} \pm 20 \%$, and a at $\lambda 808 \mathrm{~nm} \pm 10 \mathrm{~nm}$ (infrared spectrum) with fixed power of $100 \mathrm{~mW} \pm 20 \%$, according the clinical protocol of laser therapy which preconizes $0.5 \mathrm{~J}$ with red laser radiation per point on the bone exposure area and $3.0 \mathrm{~J}$ with an infrared

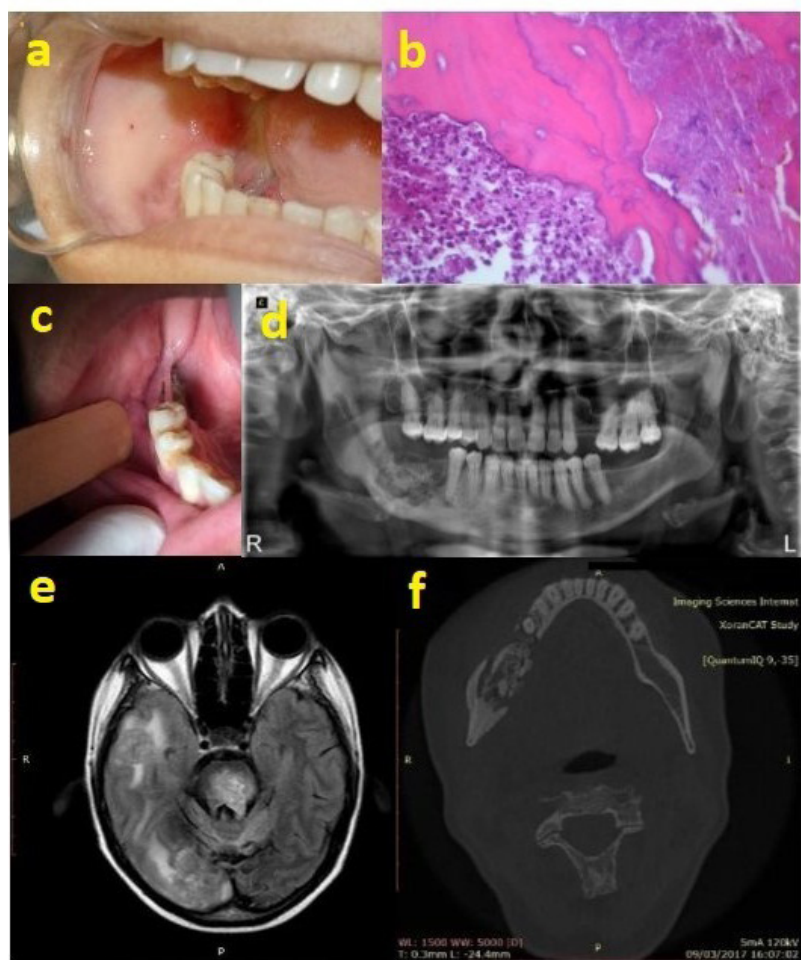

Figure 1. A sequence of image evidencing in a) Osteonecrosis of the jaw in the oral inspection; b) Histopathology analysis of the bone shows a fragment of lamellar bone tissue with gaps without osteocytes. In the periphery of bone tissue, large areas of resorption are observed; c) Evolution of nine days after the sequestrectomy; d) Panoramic radiograph showing heterogeneous image in the right body of the jaw; e) Axial cross of brain in MRI with multiple nodular lesions with intense magnetic impregnation surrounded by edema/infiltration areas distributed in the supra and infratentorial compartments, suggesting neoplastic commitment secondary at base disease; and f) Jaw cross-section in computed tomography with the presence of expansive lytic lesions with a dense sequestrum resulting in a bone-within-bone appearance.

laser per point in the buccal and lingual bone board areas. The LLLT was adopted to control swollen, therefore the lymphatic affected area was irradiated too with $2.0 \mathrm{~J}$ per point in the swollen area and $4.0 \mathrm{~J}$ near the area (Figure 2).

During the same month that the procedure was performed, the patient presented a right stroke that caused left hemiplegia, speech difficulties, and gait changes. Furthermore, in a magnetic resonance image, multiple metastases were detected (Figure 1E).

The osteonecrosis is currently stable and edema resolved; however, the exposed bone area did not regress. The chemotherapy drug was changed for capecitabine, orally. She performed chemotherapy until January (2018) with cisplatin and gemcitabine, which was suspended along with the laser therapy. Currently, she takes capecitabine and is receiving domestic palliative care due to her severe general state.

\section{Discussion}

From the case, it is possible to understand the significance of health professionals to be aware of a well-directed 


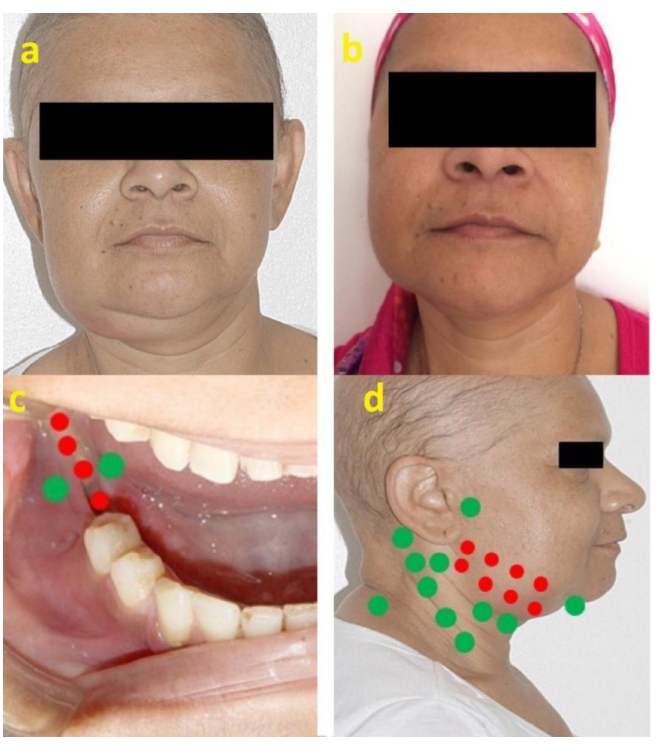

Figure 2. The evolution with the effective management showed clinical improvement (before and after the treatment, image $\mathrm{A}$ and $\mathrm{B}$, respectively) showing the adjuvant LLLT benefits in case (image $C$ and $D$, the areas which were exposed to the laser according to protocol).

anamnesis of the oncological patients with their dental demands to prevent similar cases from occurring through professional negligence.

It would be advisable to follow the preventive protocol, performing oral and clinical imaging before the beginning of treatment with BP to contain the outbreaks of infection; periodic dental appointments to eliminate possible traumatic factors; bone metabolism level monitoring; and advise patients on the risks of developing osteonecrosis. ${ }^{4}$

The LLLT provided a significant improvement regarding the signs of inflammation (mainly swollen and pain), xerostomia, bacterial control, and chemotherapyinduced oral mucositis; therefore, it is effective in MRONJ patients, avoiding oral or cutaneous fistulas and healing the mucosa on the bone tissue, and improving the life quality of the patient. ${ }^{8}$

It prevents the patient's evolution from stage 2 (exposed and necrotic bone or a fistula with evidence of infection, typically asymptomatic and symptomatic) to stage 3 (exposed and necrotic bone or fistulas with evidence of infection and with pathologic fracture or exposed necrotic bone, extraoral oral antral or oral-nasal communication, osteolysis extending to the inferior border of the mandible or sinus floor) although there was no complete healing as the extension of the injured area and poor tissue utilization during the sequestrectomy. ${ }^{1,3,7}$

\section{Ethical Considerations}

Informed consent was obtained from the patient for the publication of this report.

\section{Conflict of Interests}

The authors declare no conflict of interest.

\section{Acknowledgment}

The authors are grateful to the patient for her participation.

\section{References}

1. Ruggiero SL, Dodson TB, Fantasia J, Goodday R, Aghaloo T, Mehrotra B, et al. American association of oral and maxillofacial surgeons position paper on medicationrelated osteonecrosis of the jaw-2014 update. J Oral Maxillofac Surg. 2014;72(10):1938-1956. doi: 10.1016/j. joms.2014.04.031.

2. Ellis E III. Management of the patient undergoing radiotherapy or chemotherapy. In: Hupp JR, Tucker MR, Ellis E III. Contemporary Oral and Maxillofacial Surgery. 6th ed. St. Louis, Missouri: Mosby; 2013. p. 363-381.

3. Borromeo GL, Tsao CE, Darby IB, Ebeling PR. A review of the clinical implications of bisphosphonates in dentistry. Aust Dent J. 2011;56(1):2-9. doi:10.1111/j.18347819.2010.01283.x

4. Cortés-Motta MC, Fernández-Grisales R. Osteonecrosis de los maxilares: fisiopatología, diagnóstico y tratamiento. CES Odont. 2016;29(2):65-77. [In Spanish].

5. Campos Scarpa L, de Mello Leite LC, de Lacerda JCT, Barreto Arantes DC. Osteonecrose nos ossos da maxila e mandíbula associada ao uso do bisfosfonato de sódio. Revista Brasileira de Pesquisa em Saúde. 2010;12(1):86-92. [In Portuguese].

6. Neville BW, Damm DD, Allen CM, Chi AC. Oral and Maxillofacial Pathology. 4th ed. St. Louis, Missouri: Elsevier; 2016.

7. Latifyan S, Genot MT, Klastersky J. Bisphosphonaterelated osteonecrosis of the jaw: a review of the potential efficacy of low-level laser therapy. Support Care Cancer. 2016;24(9):3687-93. doi: 10.1007/s00520-016-3139-9.

8. Minamisako MC, Ribeiro GH, Lisboa ML, Rodríguez Cordeiro MM, Grando LJ. Medication-related osteonecrosis of jaws: A low-level laser therapy and antimicrobial photodynamic therapy case approach. Case Rep Dent. 2016;2016:6267406. doi: 10.1155/2016/6267406. 